\title{
Progress and promise in the management of chronic kidney disease
}

\section{Garabed Eknoyan MD}

$\infty$ See related review paper by Levin and colleagues, page 1154

$\mathrm{T}$ he recommendations made in this issue of $C M A J$ by Levin and colleagues ${ }^{1}$ for the management of chronic kidney disease provide clear guidance for the cooperative care of patients with chronic kidney disease by all health care providers throughout the spectrum of disease. There are specific stages and recommendations for interventions to slow disease progression, prevent complications, and reduce morbidity and mortality. This is a major paradigm shift on how kidney disease is managed, not only by nephrologists but by all health care providers and planners. In this broader context, chronic kidney disease is recognized as a heterogeneous condition whose clinical manifestations and course varies by the cause of the primary kidney disease and its severity, as well as by comorbid conditions and the rate of progressive loss of kidney function., ${ }^{2,3}$

In 1999, the Canadian Society of Nephrology published its clinical practice guidelines for the care of patients with elevated levels of serum creatinine. ${ }^{4}$ In this issue, Levin and colleagues present the new Canadian guidelines for the management of chronic kidney disease. ${ }^{1}$ A comparison of the titles, terminology and recommendations made in these 2 articles illustrates the major progress that has been made over the past decade in our understanding of kidney disease. They also illustrate how much more can now be done in the care of these patients.

Beginning with the report of patients with end-stage kidney disease by Richard Bright in 1827 and since maintenance hemodialysis became available in the 1960s, the focus of nephrology care has been the treatment and cost of end-stage renal disease. In the 1990s, it became evident that mortality among patients receiving dialysis was high. This was primarily because of comorbidities and complications of kidney disease, but also because of failure to initiate care earlier. The Canadian Society of Nephrology's guidelines ${ }^{4}$ were issued at that time. Of the 4 recommendations in these guidelines, 3 were about timely consultation and referral to a nephrologist to adequately prepare patients for dialysis or transplantation.

In the past, kidney disease was classified by its cause (e.g., pyelonephritis, glomerulonephritis, lupus nephritis). A traditional pathology-based disease classification is useful when the diagnosis determines the treatment of a specific disease. However, dialysis registry data have revealed that most referred patients had no diagnosis of kidney disease or treatment of its complications and the proportion that could be attributed to traditional pathology-based causes was relatively small and decreasing. In sharp contrast, the number of cases due to hypertension and diabetes was large and increasing, particularly among elderly patients, who accounts for most patients with end-stage renal disease. For most of these

\section{Key points}

- The definition and classification of chronic kidney disease developed in 2002 by the Kidney Disease Outcomes Quality Initiative provided a unifying approach to management of this disease.

- Emerging evidence from interventional strategies indicates that the course and outcomes of chronic kidney disease may be favourably altered.

- The Canadian Society of Nephrology's guidelines provide evidence-based recommendations for the management of chronic kidney disease.

- These guidelines provide a framework for the early detection, evaluation and shared care of patients with chronic kidney disease by all health care providers throughout the course of the disease.

patients, kidney disease had been asymptomatic, gone undetected and escaped diagnosis until well after the complications of the disease had occurred. As epidemiologic data accrued, it became increasingly evident that the systemic complications of progressive loss of kidney function (anemia, hypertension, mineral and bone disorders) were uniform and independent of the primary cause of the kidney disease. The various terms used to describe the common clinical features, such as "chronic renal failure," "pre-end stage renal disease" and "renal insufficiency," were descriptive, ill defined and vague. ${ }^{2}$ This terminology permeates the 1999 Canadian guidelines.

In 2002, the clinical practice guidelines developed by the Kidney Disease Outcomes Quality Initiative for the treatment of kidney disease provided a working definition of chronic kidney disease, irrespective of the cause of the disease. This new definition was based on the presence of either kidney damage (identified by biopsy, proteinuria or imaging studies) or a glomerular filtration rate of less than $60 \mathrm{~mL} / \mathrm{min} / 1.73 \mathrm{~m}^{2}$ for more than 3 months. ${ }^{5}$ These guidelines also proposed a classification system based on severity by the level of kidney function calculated from the estimated glomerular filtration rate. Based on the available data, the guidelines documented the increased number of complications, morbidity and mortality associated with declining glomerular filtration rates. They also describe the greater risk of death from cardiovascular disease than from progression to end-stage renal disease. These guidelines proposed a clinical action plan for the care of patients with chronic kidney disease.

Dr. Eknoyan is with the Renal Section, Department of Medicine, Baylor College of Medicine Houston, Texas, USA 
This newer terminology and classification constitutes the basis of the 2008 Canadian guidelines. ${ }^{1}$ The term "chronic kidney disease" is not even mentioned in the 1999 guidelines. In places where the vague term "elevated creatinine" was used as the determinant for referral, the new guidelines provide a specific level of kidney function (estimated glomerular filtration rate $<30 \mathrm{~mL} / \mathrm{min} / 1.73 \mathrm{~m}^{2}$ ) at which the patient should be referred. (However, primary care providers are encouraged to seek consultation at any glomerular filtration rate should the need arise.)

Some problems associated with the estimated glomerular filtration rate approach, which were clearly specified in the Kidney Disease Outcomes Quality Initiative guidelines, have been the subject of scrutiny and criticism. ${ }^{6}$ One problem is the accuracy of the formula used to estimate glomerular filtration rate and its applicability to various populations and ethnic groups of differing body size and muscle mass, and consequently creatinine production. Another problem is variability of the assays used in different clinical laboratories to measure creatinine levels, from which estimated glomerular filtration rate is derived. A worldwide effort is under way to standardize the measurement of creatinine and improve the formula to estimate glomerular filtration rate. Standardized measurements and an improved formula are anticipated to be in place by $2009 .^{7,8}$

The incidence of kidney failure is stabilizing and apparently declining, and accruing data suggest that the progressive course of chronic kidney disease is slowing., ${ }^{9,10}$ Evidence in support of interventions that account for those favourable outcomes has been used in the development and grading of the 2008 Canadian guideline recommendations. Their promise is that the adoption and application of the proposed interventions will improve outcomes. This can only be achieved with the translation of the recommendations into clinical practice, with subsequent verification by clinical performance measures and validation of these recommendations by measuring the change in outcomes after implementation. Ensuring that this promise is fulfilled and documented is the task now facing Canada's nephrologists.

Competing interests: None declared.

\section{REFERENCES}

1. Levin A, Hemmelgarn B, Culleton B, et al. Guidelines for the management of chronic kidney disease. CMAJ 2008;179:1154-62.

2. Eknoyan G. Kidney disease: wherefore, whence, and whereto? Kidney Int 2007;71:473-5.

3. Levey AS, Atkins R, Coresh J, et al. Chronic kidney disease as a global public health problem: approaches and initiatives. A position statement from Kidney Disease: Improving Global Outcomes. Kidney Int 2007;72:247-59.

4. Mendelssohn DC, Barrett BJ, Brownscombe CM, et al. Elevated levels of serum creatinine: recommendations for management and referral. CMAJ 1999;161:413-7.

5. K/DOQI clinical practice guidelines for chronic kidney disease: evaluation, classification, and stratification. Am J Kidney Dis 2002;39(Suppl 1):S1-266.

6. Eknoyan G. Chronic kidney disease definition and classification: the quest for refinements. Kidney Int 2007;72:1183-5.

7. Stevens LA, Coresh J, Levey AS. CKD in the elderly — old questions and new challenges: World Kidney Day 2008. Am J Kidney Dis 2008;51:353-7.

8. Miller WG. Reporting estimated GFR: a laboratory perspective. Am J Kidney Dis 2008;52:645-8.

9. Ruggenenti P, Remuzzi G. Kidney failure stabilizes after two-decade increase: impact on global (renal and cardiovascular) health. Clin J Am Soc Nephrol 2007;2:146-50.

10. Foley RN, Collins AJ. End-stage renal disease in the United States: An update from the United States Renal Data System. J Am Soc Nephrol 2007;18:2644-8.

Correspondence to: Dr. G. Eknoyan, Department of Medicine, 523-D, Baylor College of Medicine, One Baylor Plaza, Houston TX 77030-3498, USA; fax 713 790-0681; geknoyan@bcm.edu

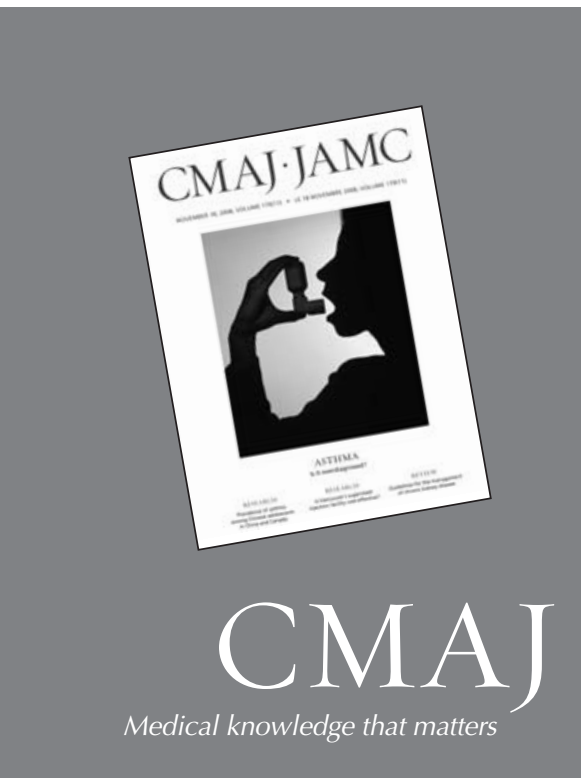

3000 volunteer reviewers from around the world are the foundation for CMAJ's thorough, criteria-based review process. The editorial staff includes scientific consultants with expertise in statistics, experimental design and epidemiology.

To order your copy, call the CMA Member Service Centre tel $888855-2555$ or $613731-8610 \times 2307$ fax 613 236-8864 cmamsc@cma.ca

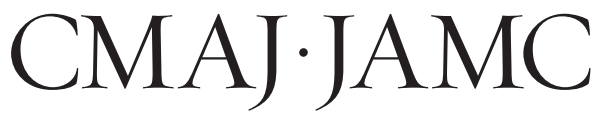

